\title{
Quotients of Abelian Surfaces
}

By

\author{
Hisao YoshiHaRA*
}

\section{§ 1. Introduction}

Let $L$ be an abelian function field of two variables over $\mathbf{C}$, and $K$ be a Galois subfield of $L$, i.e., $L$ is a finite algebraic Galois extension of $K$. We classify such $K$ by a suitable complex representation of the Galois group $G=\mathrm{Gal}(L / K)$.

Let $A$ be the abelian surface with the function field $L$. Since $g \in G$ induces an automorphism of $A$, we have a complex representation $g z=M(g) z+t(g)$, where $M(g) \in G L_{2}(\mathbf{C}), z \in \mathbb{C}^{2}$, and $t(g) \in \mathbf{C}^{2}$. Fixing the representation, we put $G_{0}=\{g \in G \mid M(g)$ is the unit matrix $\}, H=\{M(g) \mid g \in G\}$ and $H_{1}=\{M(g) \in$ $H \mid$ det $M(g)=1\}$. Then we have the following exact sequences of groups:

$$
\begin{aligned}
& 1 \rightarrow G_{0} \rightarrow G \rightarrow H \rightarrow 1, \\
& 1 \rightarrow H_{1} \rightarrow H \stackrel{d}{\rightarrow} C_{k} \rightarrow 1,
\end{aligned}
$$

where $\mathrm{d}(M(g))=\operatorname{det} M(g)$, and $H / H_{1}$ is a cyclic group $C_{k}$ of order $k \leq 12$. The quotient surface $A / G_{0}$ is also an abelian surface. Note that the function field of the surface $A / G$ is isomorphic to $K$.

Definition 1.1. We call $H$ a holonomy representation or a holonomy part of the complex representation of $G$.

The holonomy part is completely determined by Fujiki [1], in which he studies automorphisms fixing the origin. By a slightly different method from his, i.e., by considering Sylow groups of $H$, we can readily show the following.

Proposition 1.2. The order of $H$ is 5,10 or $2^{m} \cdot 3^{n}$, where $m \leq 5$ and $n \leq 2$.

Since the commutative group $G_{0}$ is a normal subgroup of $G$, the following assertion holds true.

Communicated by K. Saito, April 18, 1994.

1991 Mathematics Subject Classifications: 14K99, $14 \mathrm{~J} 15$.

* Faculty of Science, Niigata University, Niigata, 950-21 Japan. 
Corollary 1.3. The Galois group $G$ is solvable.

\section{§2. Statement of Results}

The purpose of this article is to classify $K$ by using the holonomy part and the fixed loci of $G$. But we have no suitable language in the category of fields, so we do the classification in the equivalent category, i.e., using the language of the birational classification of algebraic surfaces. Note that in the case of elliptic curve $E$ the similar classification is simple, i.e., $E / G$ is rational if and only if $H$ is not trivial.

Since the order of $G$ is finite, the quotient space $X=A / G$ is a normal algebraic surface. Let $S$ be a relatively minimal model of $X$ and $F(G)$ denote the set of fixed points of $G$. Let $[a, b]$ and $[a, b]^{*}$ denote the matrices $\left(\begin{array}{ll}a & 0 \\ 0 & b\end{array}\right)$ and $\left(\begin{array}{ll}0 & b \\ a & 0\end{array}\right)$, respectively. Let $1_{2}$ denote the unit matrix $[1,1]$. If a group is generated by $g_{1}, \ldots, g_{m}$, then it is denoted by $\left\langle g_{1}, \ldots, g_{m}\right\rangle$. Put $e_{n}=\exp (2 \pi \sqrt{-1} / n)$. The main result is stated as follows:

Theorem 2.1. We have the following classification table, where $n=2,3$, 4 or 6.

\begin{tabular}{|c|l|l|l|}
\hline$H$ & \multicolumn{3}{|c|}{ structure of $S$} \\
\hline$=\left\{1_{2}\right\}$ & \multicolumn{3}{|c|}{ abelian surface } \\
\hline$\neq\left\{1_{2}\right\},=H_{1}$ & \multicolumn{3}{|c|}{ K3 surface } \\
\hline$\neq H_{1}$ & $H=\left\langle\left[1, e_{n}\right]\right\rangle$ & $F(G)=\varnothing$ & hyperelliptic surface \\
\cline { 2 - 4 } & & $F(G)=\varnothing$ & elliptic ruled surface \\
\cline { 2 - 4 } & $\begin{array}{l}H=\langle[-1,1],[1,-1]\rangle \text { or } \\
\left\langle[-1,1]^{*},[1,-1]\right\rangle\end{array}$ & $F(G)=$ finite & Enriques surface \\
\cline { 2 - 4 } & \begin{tabular}{c} 
except the above \\
\cline { 2 - 4 }
\end{tabular} & \multicolumn{2}{|c|}{ rational surface } \\
\cline { 2 - 4 }
\end{tabular}

Since $M=M(g)$ defines an automorphism of $A$, the eigenvalues of $M$ are units of algebraic number fields of degree $\leq 4$. Then we infer easily the following from the theorem.

Corollary 2.2. If ${ }^{\#} H>24$ or the degree of the eigenvalue(s) of $M$ is 4 , then $S$ is rational.

When the degree of the eigenvalue of $M$ is $4, A$ is isogenous to $A(n)$, 
which is defined as follows (cf. [7]): let $\zeta=e_{n}, n=5,8,10$ or 12. Put

$$
\Omega_{n}=\left(\begin{array}{cccc}
1 & \zeta & \zeta^{2} & \zeta^{3} \\
1 & \zeta^{k} & \zeta^{2 k} & \zeta^{3 k}
\end{array}\right),
$$

where $k=2,3,3,5$ corresponding to each value $n$ respectively. Then $A(n)=$ $\mathrm{C}^{2} / \Omega_{n}$ is an abelian surface and $M=\left[\zeta, \zeta^{k}\right]$ defines an automorphism of $A(n)$. Furthermore in the case when $n=5, A$ is isomorphic to $A(5)$, which is a simple abelian surface and is the Jacobian variery of the curve $y^{2}=x^{5}+1$. Looking at the tables in Fujiki [1], we notice that $S$ is rational in many cases. But if $A$ is simple (and $S$ is rational), then there exists only one abelian surface $A(5)$.

Example 2.3. Note that Enriques surfaces can appear only in two holonomy representations. Such examples are as follows:

(1) Case of the Klein 4-group.

Let $A=E_{1} \times E_{2}$ and $E_{i}=\mathbf{C} /\left(1, \omega_{i}\right), \Im \omega_{i}>0$ and $G=\left\langle g_{1}, g_{2}\right\rangle$, where

$$
\left\{\begin{array}{l}
g_{1} z=[-1,-1] z \\
g_{2} z=[1,-1] z+t\left(\frac{1}{2}, \frac{1}{2}\right) .
\end{array}\right.
$$

(2) Case of the dihedral group of order 8.

Let $A$ be an abelian surface with the period matrix

$$
\left[\begin{array}{cccc}
1 & 0 & \frac{1+\omega}{2} & \frac{1-\omega}{2} \\
0 & \omega & \frac{1+\omega}{2} & \frac{1-\omega}{2}
\end{array}\right],
$$

where $\mathfrak{I} \omega>0$. Clearly $A$ is isogenous to $E \times E$, where $E=\mathbf{C} /(1, \omega)$. Let $G=\left\langle g_{1}, g_{2}\right\rangle$, where

$$
\left\{\begin{array}{l}
g_{1} z=[-1,1]^{*} z, \\
g_{2} z=[1,-1] z++^{t}\left(\frac{1}{2}, \frac{\omega}{2}\right) .
\end{array}\right.
$$

Note that $G^{\prime}=\left\langle g_{1}^{2}, g_{2}\right\rangle$ is a normal subgroup of $G$ and $A / G^{\prime}$ is also an Enriques surface with the same representation type as in (1).

Note 2.4. There is an abelian function field such that it contains each class of the function fields with Kodaira dimension 0 . In fact, let $A$ be the abelian surface defined in (2) of Example 2.3. Then we have the following diagram: 


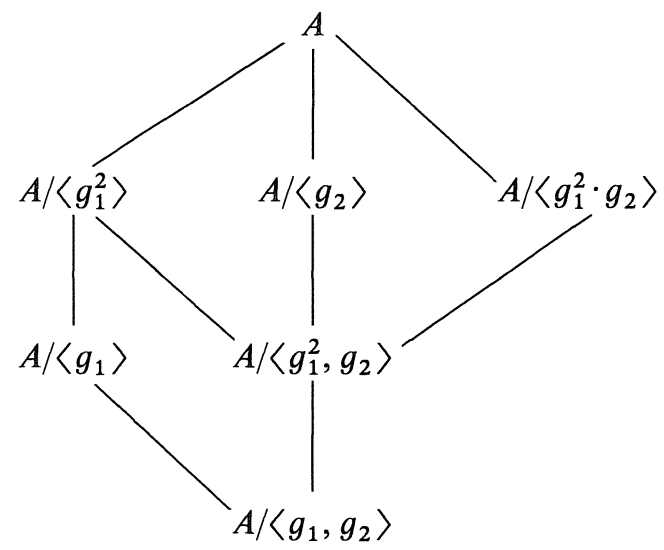

The structures of the nonsingular minimal models of the above are as follows:

$$
\begin{array}{lll}
A /\left\langle g_{1}^{2}\right\rangle, & A /\left\langle g_{1}\right\rangle, & : \mathrm{K} 3 \text { surfaces } \\
A /\left\langle g_{2}\right\rangle, & A /\left\langle g_{1}^{2} \cdot g_{2}\right\rangle, & : \text { hyperelliptic surfaces } \\
A /\left\langle g_{1}^{2}, g_{2}\right\rangle, & A /\left\langle g_{1}, g_{2}\right\rangle, & : \text { Enriques surfaces }
\end{array}
$$

Since each $M \in H$ defines also an automorphism of $A$, the quotient space $X^{\prime}=A / H$ is a normal algebraic surface. By the way, let $\tilde{Y}$ be a projective nonsingular model of an algebraic surface $Y$, then put $q(Y)=\operatorname{dim} H^{1}\left(\tilde{Y}, \mathcal{O}_{\tilde{Y}}\right)$ and $P_{m}(Y)=\operatorname{dim} H^{0}\left(\tilde{Y}, \mathcal{O}\left(m K_{\tilde{Y}}\right)\right)$, where $K_{\tilde{Y}}$ denotes the canonical divisor on $\tilde{Y}$.

Note 2.5. We have that $q(X)=q\left(X^{\prime}\right), P_{1}(X)=P_{1}\left(X^{\prime}\right)$ and $P_{m}(X) \geq P_{m}\left(X^{\prime}\right)$. In case $G_{0}$ is trivial, then $G \cong H$ as abstract groups. But in general $P_{m}(X)$ and $P_{m}\left(X^{\prime}\right)$ are distinct from each other, especially $X$ and $X^{\prime}$ are not birationally equivalent. In fact, if $H=\left\langle\left[1, e_{n}\right]\right\rangle$ and $F(G)=\varnothing$, then $X$ and $X^{\prime}$ are hyperelliptic and ruled surfaces respectively. All the $G$ which define hyperelliptic surfaces are given in Suwa [5].

If $K$ is rational, then ${ }^{\#} G \geq 3$. Note that there is only one abelian surface if $K$ is rational and ${ }^{\#} G=3$. In this case it is $E \times E$, where $E=\mathbb{C} /\left(1, e_{3}\right)$ and $H=\left\langle\left[e_{3}, e_{3}\right]\right\rangle$ (cf. [6]).

The proof of our results depends in many parts on the work of Katsura [3]. The abstract of the results above have been announced in [8], but the dihedral case of Enriques surfaces are dropped.

In the sequel we use the following notation:

$K_{S}$ : canonical divisor on $S$

$\sim$ : linear equivalence of divisors or similarity of matrices

$\Omega_{S}^{p}$ : sheaf of holomorphic $p$-forms on $S$ 


\section{§3. Proof}

First we notice that $q(S) \leq 2$ and $P_{m}(S) \leq 1$ for all $m$. Moreover $q(S)=2$ if and only if $H=\left\{1_{2}\right\}$. If $H=\left\{1_{2}\right\}$, then clearly $X=A / G$ is an abelian surface isogenous to $A$. Since $G_{0}$ is a normal subgroup of $G$, we have that $A / G \cong B / G^{\prime}$, where $B=A / G_{0}$ is an abelian surface and $G^{\prime}=G / G_{0}$. Hence we assume hereafter that $G_{0}$ is trivial and $H$ is not trivial. We enumerate several lemmas, which will be needed later.

Lemma 3.1. If $H \neq\left\{1_{2}\right\}$, then the following five conditions are equivalent:

(1) $P_{1}(S) \geq 1$,

(2) $P_{1}(S)=1$,

(3) $H=H_{1}$,

(4) $S$ is a K3 surface,

(5) $K_{S} \sim 0$.

Proof. Since $1=P_{1}(A) \geq P_{1}(S)=\operatorname{dim} H^{0}\left(A, \Omega_{A}^{2}\right)^{G}$, three conditions (1), (2) and (3) are equivalent. Owing to [1, Lemma 3.1], we see that (3) and (4) are equivalent. Hence all the conditions are equivalent.

Lemma 3.2. The irregularity $q(S)=1$ if and only if $H$ has a representation $\left\langle\left[1, e_{n}\right]\right\rangle$, where $n=2,3,4$ or 6 .

Proof. The "if part" is clear, so we prove the "only if part". Since $q=1$, there is a representation such that each element $M \in H$ is expressed as $\left(\begin{array}{ll}1 & 0 \\ c & b\end{array}\right)$. Let $a$ be the generator of $\operatorname{det} H$, and let $M_{0} \in H$ be the element such that $\operatorname{det} M_{0}=a$. Then $b=a^{k}$ for some $k \in \mathbf{N}$, i.e., $M \cdot M_{0}^{-k}=\left(\begin{array}{ll}1 & 0 \\ d & 1\end{array}\right) \in H$. Since $M \cdot M_{0}^{-k}$ has a finite order, $d$ must te 0 , i.e., $M=M_{0}^{k}$. Hence $H$ is cyclic, this means that $H$ has a representation $\langle[1, a]\rangle$, where $a=e_{n}$.

Lemma 3.3. If $M(g)$ has an eigenvalue 1 and $g$ has a fixed point, then $g$ fixes a curve $C$, i.e., $g$ is identity on $C$.

Proof. Since $M(g)$ defines an automorphism of $A, M(g)-1_{2}$ defines an endomorphism. Let $E$ be the kernel of it and $\xi$ be a fixed point of $g$, then $E+\xi$ is a desired one.

The following lemma is proved in [3, Lemmas 2.6, 2.7 and 2.8].

Lemma 3.4. Suppose that $G$ has a fixed curve or $A / G$ has a singularity which is not a rational double point. Then $P_{m}(A / G)=0$ for all $m>0$. If the Kodaira dimension of $A / G$ is 0 , then the minimal resolution of $A / G$ is the minimal model. 
Lemma 3.5. If $H$ contains an element $M$ such that $\operatorname{det} M=-1$ and ord $M \geq 3$, then $S$ is a rational surface.

Proof. Let $g \in G$ be an element satisfying $M=M(g)$. Then it is sufficient to show that $A /\langle g\rangle$ is rational. We may assume that $M=\left[a,-\frac{1}{a}\right]$, where $a \neq \pm 1$. Since det $M=-1$, the fixed points of $g$ correspond to the nonrational double points of $A /\langle g\rangle$. Since $q(A /\langle g\rangle)=0$, it is rational by Lemma 3.4 .

Now, let us prove Theorem 2.1. The proof of the case of K3 surfaces is clear from Lemma 3.1, so we treat the case $H \neq H_{1}$ hereafter. If $q(S)=1$ and $F(G)=\varnothing$, then $A$ is an unramified covering of $S$, hence $S$ is a hyperelliptic surface (cf. [5]). On the contrary, if $F(G) \neq \varnothing$, then $G$ fixes some curve by Lemmas 3.2 and 3.3. Then by Lemma $3.4, S$ is a ruled surface with $q=1$. Moreover we now show that it is also an elliptic surface. Let $\mathscr{L}$ be the lattice defining $A$, and let $h z=\left(M(g)-1_{2}\right) z$ be an endomorphism of $A$. Putting $\mathscr{L}^{\prime}=h \mathscr{L}=\left\{\left.\left(e_{n}-1\right) \ell_{2}\right|^{t}\left(\ell_{1}, \ell_{2}\right) \in \mathscr{L}\right\}$, we see that $E=\mathbf{C} / \mathscr{L}^{\prime}$ is an elliptic curve with an automorphism defined by a multiplication $e_{n}$. Since each irreducible component of fixed curves of $G$ is a nonsingular elliptic curve and they are disjoint, the surface $A / G$ is smooth. Cleary there are no $(-1)$-curves on $A / G$, hence $S=A / G$. Thus we have a morphism $\varphi: S \rightarrow E /\left\langle e_{n}\right\rangle \cong \mathbb{P}^{1}$. By the Stein factorization theorem we have a fiber space $\tilde{\varphi}: S \rightarrow C$, whose general fiber is an elliptic curve. Moreover we infer from the canonical bundle formula for an elliptic surface that $C \cong \mathbb{P}^{1}$.

Finally we treat the last case. The surfaces not considered above satisfy that $P_{1}=q=0$, hence they are Enriques or rational surfaces. Hereafter we assume that $S$ is an Enriques surface. Then we get the following assertions from the inequality $1=P_{2}(S) \leq \operatorname{dim} H^{0}\left(A,\left(\Omega^{2}\right)^{\otimes 2}\right)^{G}$ :

(i) $\operatorname{det} M= \pm 1$ for each $M \in H$,

(ii) there is an $M \in H$ such that $\operatorname{det} M=-1$.

First we show the following:

Claim 1. $H$ is a 2-group, i.e., ${ }^{\#} H=2^{m}$.

Proof. Suppose that $n=2$ in Proposition 1.2. Then the 3-Sylow group of $H$ has a representation $\left\langle\left[1, e_{3}\right],\left[e_{3}, 1\right]\right\rangle$, because it has no irreducible representation of degree 2. This contradicts (i) above. Next suppose that $n=1$. Then there are two elements $M\left(g_{1}\right), M\left(g_{2}\right) \in H$ such that $M\left(g_{1}\right) \sim$ $\left[e_{3}, e_{3}^{2}\right]$ and $M\left(g_{2}\right) \sim[1,-1]$ by Lemma 3.5. So that we have a representation as $M\left(g_{2}\right)=[1,-1]$ and $M\left(g_{1}\right)=\left[\begin{array}{rr}-\frac{1}{2} & b \\ a & -\frac{1}{2}\end{array}\right]$, where $a b=-\frac{3}{4}$, since 
$M\left(g_{1}\right) \cdot[1,-1] \sim[1,-1]$. Let $g_{2} z=[1,-1] z+{ }^{t}\left(c_{1}, c_{2}\right)$. Since $g_{2}^{2}$ is an identity, we have that $t\left(2 c_{1}, 0\right) \in \mathscr{L}$. Moreover since $M\left(g_{1}\right)$ defines an automorphism of $A$, we get that $M\left(g_{1}\right) \cdot{ }^{t}\left(2 c_{1}, 0\right) \in \mathscr{L}$. Hence we have that ${ }^{t}\left(c_{1},-2 a c_{1}\right) \in \mathscr{L}$, this implies that $F\left(g_{2}\right) \neq \varnothing$. By Lemmas 3.3 and $3.4, A / G$ is rational, which is a contradiction. Therefore $H$ is a 2-group.

We will complete the proof of the theorem by examining the following cases separately:

Case (1): $H$ is commutative.

Case (2): $H$ is not commutative.

First we consider the case (1). Suppose that there is an element $M$ with ord $M \geq 3$. Then by Lemma 3.5 , det $M=1$, moreover ord $M=4$ by Claim 1 and Lemma 3.5. In fact, if ord $M=8$, then $\operatorname{det} M=-1$. Hence there are two elements $\left[e_{4},-e_{4}\right]$ and $[1,-1]$, since $H$ is commutative. Then there is an element $\left[e_{4}, e_{4}\right] \in H$, which is a contradiction by Lemma 3.5. Therefore $H$ is generated by $[-1,1]$ and $[1,-1]$.

Next we consider the case (2). Note that the holonomy representation $H$ is irreducible, because $H$ is not commutative. Since $H$ is a 2-group and the element of order 2 in $H_{1}$ is unique, i.e., it is $[-1,-1]$, the subgroup $H_{1}$ is one of the following (cf. [2, Theorem 12.5.2]):

(2-1) a cyclic group,

(2-2) the quarternion group.

First we prove that the case (2-2) cannot occur. Suppose that $H_{1}$ corresponds to this case. Then, we may assume that $H_{1}=\left\langle M_{1}, M_{2}\right\rangle$, where ord $M_{i}$ $=4(\mathrm{i}=1,2)$. We take a representation such that $M=[1,-1] \in H$. Then, since $M_{i} \cdot M \sim[1,-1]$ by Lemma 3.5 , we obtain that $M_{i}=\left[a_{i}, b_{i}\right]^{*}$, where $a_{i} b_{i}=-1(\mathrm{i}=1,2)$. Since $M_{2} \cdot M_{1}=M_{1}^{3} \cdot M_{2}$, we can write $M_{2}=\left[e_{4},-e_{4}\right]$. $M_{1}$. Hence $H_{1} \ni\left[e_{4},-e_{4}\right]$, i.e., $H \ni\left[e_{4}, e_{4}\right]$. This is a contradiction by Lemma 3.5. Therefore $H_{1}$ must be a cyclic group and ${ }^{\#} H_{1}=2$ or 4 , which means that ${ }^{\#} H=4$ or 8 . Since $H$ is not commutative, we have that ${ }^{\#} H=8$. Let $M_{1}$ be a generator of $H_{1}$. Then $H$ is generated by $M_{1}$ and $M_{2}=[1,-1]$. Since $M_{1} \cdot M_{2} \sim M_{2}$, we have that $M_{1}=[a, b]^{*}$, where $a b=-1$. Thus $H$ is a dihedral group of order 8 , which has an equivalent representation: $M_{1}=$ $[-1,1]^{*}$ and $M_{2}=[1,-1]$. Consequently $H$ has a representation $\langle[-1,1]$, $[1,-1]\rangle$ or $\left\langle[-1,1]^{*},[1,-1]\right\rangle$. Since $H \ni[-1,-1], F(G)$ is a non-empty finite set.

Lemma 3.6. The surface $S$ is an Enriques surface if and only if the following conditions are satisfied:

(a) $H$ has a representation $\langle[-1,1],[1,-1]\rangle$ or $\left\langle[-1,1]^{*},[1,-1]\right\rangle$,

(b) ${ }^{\#} F(G)<\infty$.

Proof. We have proved the "only if part", so we prove the "if part". By taking a suitable basis, we may assume in the latter case of (a) that $G=\left\langle g_{1}, g_{2}\right\rangle$, 
such that

$$
\left\{\begin{array}{l}
g_{1} z=[-1,1]^{*} z \\
g_{2} z=[1,-1] z+c,
\end{array}\right.
$$

where $z={ }^{t}\left(z_{1}, z_{2}\right)$ and $c={ }^{t}\left(c_{1}, c_{2}\right)$. Since we have assumed that $G_{0}$ is trivial, we have that $g_{2}^{2} z=z$. Hence ${ }^{t}\left(2 c_{1}, 0\right) \in \mathscr{L}$, where $\mathscr{L}$ is the lattice. Putting $G_{1}=\left\langle g_{1}^{2}\right\rangle, G_{2}=\left\langle g_{1}\right\rangle$ and $G_{3}=\left\langle g_{1}^{2}, g_{2}\right\rangle$, which are normal subgroups of $G$, we have normal algebraic surfaces $X_{i}=A / G_{i}(i=1,2,3)$. Let $S_{i}$ be a relatively minimal model of $X_{i}$. By definition $S_{1}$ is a Kummer surface. The $g_{2}$ induces an automorphism $\bar{g}_{2}$ of $X_{1}$, which permutes the singular points of $X_{1}$. Note that $\bar{g}_{2}$ has no fixed points, otherwise there exists $\xi \in A$ satisfying $g_{1}^{2} g_{2} \xi=\xi$. Then $g_{1}^{2} g_{2}$ fixes a curve by Lemma 3.3, which contradicts (b). Since the singular points of $X_{1}$ are only rational double ones of type $\mathrm{A}_{1}, \bar{g}_{2}$ can be extended to an automorphism $\varphi_{2}$ of $S_{1}$. Therefore $S_{1} / \varphi_{2}$ is an Enriques surface, since $\varphi_{2}$ has no fixed points. It is clear from Lemma 3.4 that $S_{1} / \varphi_{2} \cong S_{3}$. The group $G$ whose holonomy part is the Klein 4-group is regarded as $G_{3}$, so we have proved the former case of (a).

Note that $F\left(g_{1}\right) \subset F\left(g_{1}^{2}\right),{ }^{\#} F\left(g_{1}\right)=4$ and ${ }^{\#} F\left(g_{1}^{2}\right)=16$. Of course $g_{2}\left(F\left(g_{1}^{2}\right)\right)$ $=F\left(g_{1}^{2}\right)$ and $g_{2}\left(F\left(g_{1}\right)\right)=F\left(g_{1}\right)$.

Claim 2. If $\xi \in F\left(g_{1}^{2}\right)$, then $g_{2} \xi \neq g_{1} \xi$ and $g_{2} \xi \neq g_{1}^{2} \xi$.

Proof. Suppose the contrary. Then $g_{2} g_{1} \xi=\xi$ or $g_{2} g_{1}^{2} \xi=\xi$, since $g_{1}^{2} \xi=$ $\xi$. By Lemma 3.3, $F(G)$ contains a curve, which is a contradiction.

By this claim $g_{2}$ induces an automorphism $\hat{g}_{2}$ of $X_{2}$, which permutes the singular points of $X_{2}$. Clearly 6 [resp. 4] pieces of the singular points of $X_{2}$ are rational singularities of type $A_{1}$ [resp. $\left.A_{3}\right]$.

Claim 3. The automorphism $\hat{g}_{2}$ of $X_{2}$ can be extended to one $\psi$ of $S_{2}$.

Proof. It is sufficient to consider $\hat{g}_{2}$ near the singular points $P$ and $P^{\prime}$ of type $A_{3}$. Since these singularities are rational double ones, $\hat{g}_{2}$ can be extended to an automorphism of $S_{2}$. In another way, we can show this directly as follows. Letting $\left(x_{1}, x_{2}\right)$ and $\left(x_{1}^{\prime}, x_{2}^{\prime}\right)$ are local coordinates of $P$ and $P^{\prime}$ respectively, we can express as $\hat{g}_{2}\left(x_{1}, x_{2}\right)=\left(x_{1}^{\prime}, x_{2}^{\prime}\right)$, where $x_{1}^{\prime}=x_{2}$ and $x_{2}^{\prime}=$ $-x_{1}$. Each singularity is isomorphic to one defined by $y_{2}^{4}=y_{1} y_{3}$. Such a singularity is resolved by $M(2,2,2)$, (see, [4, Ch. II]). Expressing $M(2,2,2)$ by local coordinates, we infer that locally $\hat{g}_{2}$ can be extended to an isomorphism between $M(2,2,2)$. The minimal resolution of $X_{2}$ coincides with the minimal model of it by Lemma 3.4 , so $\psi$ is an automorphism of $S_{2}$.

The $\psi$ has no fixed points and has order 2. Therefore $S_{2} / \psi$ is an Enriques surface, which coincides with $S$. Thus we complete the proof of Theorem 
2.1. Since ${ }^{\#} H_{1} \leq 24$ (see the next Note 3.7 ), Corollary 2.2 is easily deduced from Theorem 2.1. For the proof that the surfaces in Example 2.3 are Enriques surfaces, it is sufficient to check ${ }^{\#} F(G)<\infty$ by Lemma 3.6, i.e., $F(g)=\varnothing$ if $\operatorname{det} M(g)=-1$.

Finally we mention the structure of complex tori with the automorphism $H=H_{1} \neq\left\{ \pm 1_{2}\right\}$.

Note 3.7. Let $T$ be a complex torus of dimension 2. Suppose that $T$ has a finite automorphism group $G$ satisfying $H=H_{1} \neq\left\{ \pm 1_{2}\right\}$. Then $H$ is isomorphic to one of the following:

(1) cyclic group of order $n=3,4$ or 6 .

(2) binary dihedral group $(2,2,2)$ or $(2,2,3)$, or binary tetrahedral group $(2,3,3)$.

In the case (1), $T$ is isomorphic to $\mathrm{C}^{2} / \Omega$ such that

$$
\Omega=\left(\begin{array}{cccc}
1 & 0 & x & y \\
0 & 1 & -y & x+k y
\end{array}\right),
$$

where $(x, y) \in \mathbf{C}^{2}-\mathbf{R}^{2}$ and $k=-1,0,1$, according to $n=3,4,6$, respectively. In this case $H$ is generated by $M_{k}=\left(\begin{array}{rr}0 & 1 \\ -1 & k\end{array}\right)$. We can show, by considering the Riemann conditions, that $T$ is not algebraic if $x$ and $y$ do not satisfy equations $f(X, Y)=0$, where $f(X, Y) \in \mathbf{Q}[X, Y]$ and $\operatorname{deg} f \leq 2$. Putting $y=0$, we see that, for any elliptic curve $E$, the abelian surface $A=E \times$ $E$ admits the action $M_{k}$. In the case (2), $\mathrm{T}$ is not an algebraic surface or a singular abelian surface, see Fujiki [1, Proposition 3.7 and Lemma 5.6].

\section{References}

[1] Fujiki, A., Finite automorphism groups of complex tori of dimension two, Publ. RIMS, Kyoto Univ., 24 (1988), 1-97.

[2] Hall, M., The theory of groups, Macmillan 1959.

[3] Katsura, T., Generalized Kummer surfaces and their unirationality in characteristic $p, J$. Fac. Sci. Univ. Tokyo, Sect. IA, 34 (1987), 1-41.

[4] Laufer, H. B., Normal two-dimensional singularities, Ann. of Math. Stud., 71, Princeton Univ. Press 1971.

[ 5] Suwa, T., On hyperelliptic surfaces, J. Fac. Sci. Univ. Tokyo, Sect. I, XVI (1970), 469-476.

[6] Tokunaga, H. and Yoshihara, H., Degree of irrationality of abelian surfaces, to appear in J. Algebra.

[7] Yoshihara, H., Structure of complex tori with the automorphisms of maximal degree, Tsukuba J. Math., 4 (1980), 303-311.

[8] - Galois subfields of abelian functon field of two variables, Proc. Japan Acad., 70 (1994), 3-5. 
